\title{
Re: Comparing Open Radical Cystectomy and Robot-Assisted Laparoscopic Radical Cystectomy: A Randomized Clinical Trial
}

\author{
Bochner B. H1, Dalbagni G1, Sjoberg D. D2, Silberstein J1,3, Keren Paz GE1, Donat S. M1, \\ Coleman J. A1, Mathew S1, Vickers A2, Schnorr G. C2, Feuerstein M. A1, Rapkin B4, Parra R. O1, \\ Herr H. W1, Laudone V. P1
}

1 Memorial Sloan Kettering Cancer Center, Urology Service, Department of Surgery, New York, USA

2 Memorial Sloan Kettering Cancer Center, Department of Epidemiology and Biostatistics, New York, USA

3Tulane University Faculty of Medicine, Department of Urology, Los Angeles, USA

${ }_{4}^{4}$ Albert Einstein College of Medicine, Department of Epidemiology and Population Health, New York, USA

Eur Urol 2015;67:1042-1050. doi: 10.1016/j.eururo.2014.11.043. Epub 2014 Dec 8.

\section{EDITORIAL COMMENT}

In this prospective randomized trial, 118 patients were randomized to open radical cystectomy (ORC) and robot-assisted laparoscopic radical cystectomy (RALRC). All patients underwent open urinary diversion. The authors compared overall 90-days complications, surgical indices, such as estimated blood loss, operative time, pathologic outcomes, 3- and 6-mo patient-reported quality-of-life ( $\mathrm{QO} \mathrm{L})$ outcomes, and total operative room and inpatient costs. There were also no differences in pathological outcomes, hospital stay or quality of life measures at 3 and 6 months. The robotic group had an advantage of less blood loss but had longer operative times and increased cost.

However, the study had some concerns that are not sufficiently powerful to conclude that these two techniques were equivalent.

1- In oncological perspective, only 8.5\% of RARC patients and 8.8\% of ORC patients were staged with T3/T4 disease, and 58\% of RARC patients and 55\% of ORC patients had non-muscle-invasive disease (pTo/1 disease). That means this study did not represent all cystectomy patients. Additionally, only $17 \%$ of patients had positive lymph nodes that show us selection bias towards good risk patients. This bias might affect pathological outcomes.

2- The rate of postoperative complications was the primary end point of this study because complications occur in approximately two-thirds of patients undergoing radical cystectomy and orthotopic diversion. In this study, $21 \%$ of all patients experienced high-grade complications. Infectious complications (grade 2-5) occurred in $38 \%$ of RARC and $29 \%$ of ORC patients. Cardiac and gastrointestinal grade 2-5 complications were observed in 38\% of RARC and $43 \%$ of ORC patients. These results let us think that RALRC was not superior to ORC.

As a conclusion, this study shows that in a carefully selected, rather low-risk group of patients, RARC does not substantially improve outcomes versus ORC. Additionally, this study does not give any data about the long-term functional outcomes, such as preservation of sexual and renal function or the incidence of perfectly functioning orthotopic bladder substitutes, which is most important for patients. As a backword, it is the surgeon that makes the difference, not the instrument used. 